\title{
EL PROFESORADO UNIVERSITARIO: SU CONCEPCIÓN \\ Y FORMACIÓN COMO MODELO DE ACTUACIÓN ÉTICA \\ Y PROFESIONAL
}

\author{
Viviana González Maura \\ Universidad de La Habana, Cuba
}

\section{INTRODUCCIÓN}

La Educación Superior Contemporánea tiene la misión de "formar profesionales altamente capacitados que actúen como ciudadanos responsables, competentes y comprometidos con el desarrollo social" (UNESCO, 1998), misión que no es posible cumplir desde los postulados de una enseñanza tradicional que centra la atención en el profesor como tansmisor de conocimientos y valores que son reproducidos por los estudiantes de forma acrítica y descontextualizada de la práctica profesional.

La formación del ciudadano responsable, competente y comprometido que la sociedad actual necesita, es sólo posible desde una nueva concepción del profesor como persona que acompaña al estudiante en el proceso de construcción de conocimientos, actitudes y valores, en el que asume nuevas funciones que se expresan en su condición de gestor de información, guía del proceso de aprendizaje de sus estudiantes y que se resumen en su condición de modelo educativo. (Martínez, Buxarrais y Esteban, 2002).

El propósito de este artículo es compartir algunas reflexiones y experiencias de la autora en torno a la concepción y formación del docente universitario como modelo educativo potenciador del desarrollo moral y profesional de sus estudiantes.

- ¿Cuáles son las características esenciales del profesor como modelo educativo?

- ¿Qué significa entender la formación del profesor como proceso de desarrollo profesional?

- ¿Cómo concebir un programa de formación postgraduada potenciador del desarrollo profesional de los profesores universitarios?

A lo largo de este artículo intentaremos ofrecer algunas respuestas a estos interrogantes.

\section{EL PROFESOR COMO MODELO EDUCATIVO.}

La concepción del profesor como persona que acompaña al estudiante en el proceso de enseñanzaaprendizaje exige nuevas funciones que se expresan en la consideración del profesor como modelo educativo.

\section{El profesor como gestor de información.}

El profesor universitario actual ha de ser un experto en su materia que manifieste ante todo dominio de los conocimientos de la asignatura o área de conocimiento que trabaja. La diferencia en la experticia del profesor actual con relación al tradicional se manifiesta en la posibilidad de ser un gestor de información y no un transmisor de conocimientos acabados como verdades absolutas, en tanto comparte 
sus conocimientos, vivencias, experiencias, y reflexiones con sus alumnos, respecto a los contenidos de enseñanza, en un ambiente democrático que los motiva a profundizar en el estudio y les orienta, no sólo sobre las fuentes bibliográficas a utilizar, sino también cómo y dónde procurarlas.

\section{El profesor como guía del proceso de enseñanza-aprendizaje.}

No basta con que el profesor sea un conocedor de su materia, también debe tener la formación pedagógica necesaria que le posibilite utilizar metodologías de enseñanza-aprendizaje que orienten al estudiante en la construcción de sus conocimientos, actitudes y valores. Es en este sentido que hablamos del profesor universitario como orientador, mediador en el aprendizaje de sus estudiantes (González Maura 1999, 2002 ), para ello el profesor debe diseñar situaciones y tareas de aprendizaje, de contenido profesional, que potencien tanto el desarrollo de conocimientos y habilidades como de las dimensiones de la personalidad moral del estudiante que le permitan un aprendizaje profesional ético, una formación integral como persona que ejerce una profesión. Para que el profesor sea un orientador, un guía en el aprendizaje de sus estudiantes debe ser auténtico en sus relaciones, empático, lograr comprensión crítica, saber escuchar a sus alumnos, asumirlos como sujetos de aprendizaje, como personas en formación, con sus virtudes y defectos, aceptarlos tal y como son, ha de ser tolerante, y al mismo tiempo, propiciar en sus estudiantes el desarrollo de todas estas cualidades en el proceso de enseñanza-aprendizaje. Para este fin, son muy útiles las metodologías de aprendizaje participativo que posibilitan al estudiante asumir un papel protagónico en la construcción de conocimientos y valores en un ambiente dialógico, de respeto y tolerancia a las diferencias, bajo la guía del profesor. ( Navarro, 2000, Vinuesa, 2002, Brockbank, Gill, 2002, Escamez, 2001)

Para lograr ser un guía del aprendizaje es importante que el profesor maneje acertadamente la dialéctica de la directividad-no directividad del proceso de enseñanzaaprendizaje, que Paulo Freire expresa como relación entre la libertad y la autoridad. En este sentido considero que el profesor es directivo en tanto que es la persona experta que tiene los conocimientos, la motivación y los recursos pedagógicos para guiar el aprendizaje del estudiante, pero su directividad ha de ser flexible de manera que de espacio al protagonismo y participación de los estudiantes, sólo así es posible potenciar el desarrollo del estudiante como sujeto de aprendizaje.

Freire en su libro Pedagogía de la Autonomía al respecto expresa: "Como profesor tanto lidio con mi libertad como con mi autoridad en ejercicio, pero también lidio directamente con la libertad de los educandos, que debo respetar y con la creación de su autonomía tanto como con los ensayos de construcción de la autoridad de los educandos".Freire (1998:92)

\section{El profesor como modelo de actuación ética y profesional.}

Para lograr cumplir las funciones anteriores el profesor universitario ha de ser para sus estudiantes un modelo de actuación personal y profesional, un ejemplo que estimule a sus estudiantes en el proceso de su construcción como persona en el ejercicio de la profesión. No es posible ser modelo de actuación para sus alumnos si el profesor no ha logrado un desarrollo profesional que se exprese en su motivación por la profesión, su dedicación y entrega al trabajo, su condición de experto en el área de conocimientos que trabaja y su formación psicopedagógica que le posibilite establecer un proceso de comunicación con sus estudiantes sustentado en el diálogo. Pero además, ser modelo de actuación implica necesariamente el desarrollo del profesor como persona moral. Para ser modelo hay que ser coherente, tiene que mostrar 
correspondencia entre lo que dice y hace, tiene que expresar vocación y compromiso con la educación, sólo así puede ser un ejemplo para sus alumnos. (Martínez, 2000), (Buxarrais, 1997)

Como decía anteriormente, la asunción de estas funciones es sólo posible desde una concepción del profesor como persona que se construye en el ejercicio de la docencia en un proceso permanente y continuo de desarrollo que le conduce a la autonomía moral y profesional. (Imbernon, 1994, 1999, 2001, 2002), (Freire, 1998)

Al respecto Freire expresa: "En el fondo la relación entre educador y educando, entre autoridad y libertades, entre padres, madres, hijos e hijas, es la reinvención del ser humano en el aprendizaje de su autonomía."

"Me muevo como educador porque primero, me muevo como persona" (1998: 91)

\section{FORMACIÓN Y DESARROLLO PROFESIONAL DEL PROFESOR UNIVERSITARIO.}

Durante mucho tiempo bajo el manto de la Pedagogía tradicional predominó la perspectiva instrumentalista de la formación del profesorado que centró su atención en la formación de conocimientos y habilidades para el ejercicio de una docencia que privilegia la transmisión y reproducción de conocimientos, al margen de las cualidades personales del profesor y de los estudiantes. En la actualidad ante los reclamos de las tendencias pedagógicas contemporáneas que cambia la mirada de la enseñanza y sus resultados, al aprendizaje en tanto proceso de construcción de conocimientos y valores en condiciones de interacción social, la atención se centra en el profesor y el estudiante como sujetos de enseñanza y aprendizaje y, por tanto, en la formación del profesorado desde una perspectiva humanista, como proceso de desarrollo de la personalidad del profesor en el ejercicio de la docencia.

La formación del profesorado desde una perspectiva humanista se sustenta en la concepción del profesor como persona y por tanto, en la necesidad de potenciar a través de la educación, el desarrollo profesional del profesor como dimensión de su desarrollo personal.

En las investigaciones que hemos desarrollado en torno a la formación del profesorado universitario para la educación moral y profesional del estudiante, desde una perspectiva humanista, hemos concebido el desarrollo profesional del profesorado como: "Un proceso permanente, continuo y gradual de tránsito hacia la autodeterminación en é ejercicio de la docencia, que implica necesariamente la reflexión crítica y comprometida del profesor con la transformación de la práctica educativa y la calidad de su desempeño, en un ambiente diálogico y participativo, en el contexto histórico-concreto de su actuación profesional." (González Maura et al, 2003a)

El desarrollo profesional que conduce a la autodeterminación profesional y ética del profesor en su desempeño, es un proceso permanente en tanto se produce a lo largo de la vida profesional, continuo porque tiene lugar de forma ininterrumpida en el ejercicio de la docencia y gradual porque transita progresivamente hacia la autonomía profesional.

Un elemento esencial, que posibilita el tránsito hacia la autodeterminación profesional del docente universitario, lo constituye la reflexión crítica y comprometida sobre su práctica educativa, de ahí la importancia de potenciar, a través de los programas de formación docente la reflexión del docente y su 
compromiso con el mejoramiento de la calidad de su práctica profesional. (Canfux, 2000), (González Maura, Blández, Sierra, 2003)(González Maura 2003a)(Martín et al 2002)

El espacio de aprendizaje que permite potenciar el desarrollo de la reflexión crítica y comprometida del docente sobre el ejercicio de su profesión, es el diálogo y la participación, por lo que la metodología participativa y el trabajo grupal constituyen escenarios importantes para el desarrollo profesional en los programas de formación docente. (Freire, 1998). (Hoyos, 1999), (Navarro, 2000), (Vinuesa, 2002), (Cortina, 1999), (Kraftchenko y Segarte, 1999)

Las investigaciones que hemos realizado acerca del desarrollo profesional de profesores universitarios en el ejercicio de una docencia dirigida a potenciar el desarrollo moral y pofesional del estudiante (González Maura 1999, 2002, 2002ª , 2003, 2004a) nos han permitido precisar un conjunto de indicadores del desarrollo profesional del profesor que apuntan hacia una actuación profesional autodeterminada, ellos son:

- Interés profesional.

- Satisfacción en el desempeño profesional.

- Reflexión crítica en el desempeño profesional.

- Perseverancia en la actuación profesional.

- Compromiso con la calidad del desempeño profesional.

- Flexibilidad en la actuación profesional.

- Tendencia al autoperfeccionamiento profesional.

- Dominio de conocimientos y habilidades profesionales.

- Capacidad dialógica.

- Actuación profesional ética.

Estos indicadores se integran en la actuación profesional del profesor que constituye para sus estudiantes un modelo de actuación ética y profesional.

Si partimos de la necesidad de considerar entre las nuevas funciones del profesor universitario, la función educativa, expresada en su condición de guía y modelo en la educación moral y profesional de sus estudiantes y del conocimiento de que el profesor universitario es un profesional que no ha recibido formación psicopedagógica para el ejercicio de la docencia en sus estudios de pregrado, estaremos de acuerdo en que la formación psicopedagógica del docente universitario constituye un componente esencial de su formación postgraduada.

La formación postgraduada del profesor universitario, por tanto, ha de contemplar entre sus objetivos esenciales, la formación para un ejercicio responsable, ético y competente de la docencia, que se exprese en la autonomía y el compromiso con la calidad de su función educativa, objetivo que se logra en la medida en que el programa de formación postgraduada se diseñe, ejecute y evalúe teniendo en cuenta su contribución al desarrollo profesional del docente. (González 2003루 , 2004b) 


\section{PRINCIPIOS DE UN PROGRAMA DE FORMACIÓN POSTGRADUADA QUE} TRIBUTE AL DESARROLLO PROFESIONAL DEL PROFESOR UNIVERSITARIO

Entre los principios a tener en cuenta en el diseño de un programa de formación postgraduada para docentes universitarios que tribute a su desarrollo profesional se destacan:

\section{El reconocimiento del profesor universitario como sujeto de su desarrollo profesional}

En virtud de este principio se reconoce la necesidad de que el docente asuma una postura comprometida y responsable en su desarrollo profesional a través del programa. Para ello el programa de formación debe potenciar el desarrollo integral del profesor como persona, a través del reconocimiento de la necesaria unidad del pensar, el sentir y el actuar del profesor en el ejercicio de la docencia, es decir, de sus conocimientos, habilidades, actitudes, motivos y valores en la regulación de su actuación profesional.

El reconocimiento del carácter orientador del programa en el proceso de formación profesional

El programa de formación profesional ha de tener un carácter orientador, ello implica que los coordinadores de los programas de postgrado asuman una función tutorial en el desarrollo profesional de los docentes que los conduzca de forma gradual hacia el desarrollo de la autonomía en el ejercicio de la profesión.

\section{La necesaria integración de la teoría y la práctica en el proceso de formación profesional}

Todas las modalidades de un programa de formación postgraduada que tribute al desarrollo profesional del docente exigen la integración de la teoría y la práctica profesional y su contextualización en el desempeño profesional del docente a través de la investigación científica.

La concepción de la formación permanente como tributaria de un desarrollo profesional tendiente a la autonomía y el compromiso con la calidad del desempeño profesional, a través de la vinculación de la teoría y la práctica profesional, es referida por Imbernon cuando plantea los 5 pilares o principios en que considera debe sustentarse la formación permanente:

- Aprender continuamente de forma colaborativa, participativa.

- Conectar los conocimientos producto de la socialización vulgar con nuevas informaciones en un proceso coherente de formación (adecuación de las modalidades a la finalidad formativa) para rechazar o aceptar los conocimientos en función del contexto.

- Aprender mediante la reflexión individual y colectiva en la resolución de situaciones problemáticas de la práctica.

- Aprender en un ambiente formativo de colaboración y de interacción social.

- Elaborar proyectos de trabajo conjunto y vincularlos a la formación mediante estrategias de investigación-acción. (2001: 40)

\section{LA INVESTIGACIÓN-ACCIÓN COMO POTENCIADORA DEL DESARROLLO PROFESIONAL DEL PROFESOR UNIVERSITARIO.}

Los estudios acerca de la formación docente como desarrollo profesional (Imbernon 1994, 1999, 2002) han constatado la importancia de la investigación como elemento potenciador de la autonomía del 
profesor en el ejercicio de su profesión. (Muñoz, Quintero y Munevar, 2002), (Orozco, 2000), (Rodríguez, 1999), (Sánchez, 2002), (Hernández, 2000)

La investigación educativa (Elliot 1993), en tanto se orienta a la participación activa y comprometida del docente en la identificación y búsqueda de vías para la solución científica de problemas de la práctica profesional, constituye un recurso de inestimable valor para el desarrollo profesional del docente universitario en el proceso de su formación profesional. Es por ello que deviene eje transversal de los programas de formación postgraduada. (González Maura, 2004b)

La investigación educativa y, en particular, la investigación-acción (Kemmis y Mc Taggart, 1992) permite a través del vínculo de la teoría y la práctica profesional potenciar el protagonismo del docente en la búsqueda de alternativas de solución a los problemas que enfrenta en su desempeño profesional y constituye una vía importante para potenciar la reflexión crítica del docente sobre su práctica profesional.

Independientemente de las modalidades que pueda asumir un programa de postgrado para la formación docente: taller, curso, entrenamiento, diplomado, maestría, doctorado, ha de tener en cuenta necesariamente la investigación como recurso de aprendizaje para el desarrollo profesional del docente en la consideración de los siguientes aspectos:

- Asunción de una postura reflexiva y crítica en el desempeño profesional.

- Identificación de problemas de la práctica profesional.

- Búsqueda individual y colectiva de alternativas de solución a los problemas detectados.

- Aplicación de alternativas de solución.

- Análisis y valoración individual y grupal de los resultados de las alternativas aplicadas en la solución ética y científica de los problemas identificados y de su influencia en el desarrollo profesional del docente.

- Ajuste y perfeccionamiento de las alternativas aplicadas y elaboración de proyectos individuales y grupales para el desarrollo profesional.

En las investigaciones que estamos desarrollando acerca de la educación de valores desde el curriculum universitario (Ojalvo, et.al 2003) hemos trabajado la investigación-acción como modalidad de formación postgraduada en la preparación de profesores universitarios para el diseño, aplicación y evaluación de estrategias de educación en valores desde sus asignaturas. (González Maura 2003a). En particular hemos trabajado la variante de investigación-acción-colaborativa (Pine, 1981) y hemos podido constatar sus posibilidades como potenciadora del desarrollo profesional del docente.

La investigación-acción-colaborativa constituye una alternativa de desarrollo profesional de los docentes a través de la investigación en tanto posibilita el desarrollo de actitudes, valores y habilidades profesionales en los docentes a partir de su participación activa y comprometida en la búsqueda de soluciones a los problemas educativos identificados en la práctica profesional.

La investigación-acción-colaborativa permite a los docentes transitar gradualmente, bajo la orientación de un coordinador, hacia un nivel superior de participación, la participación plena, que se manifiesta en la autogestión en la que el propio grupo de profesores determina sus objetivos, escoge sus medios y establece los controles pertinentes en el desarrollo de la tarea educativa. 
La investigación-acción-colaborativa constituye una vía para el desarrollo profesional del profesor universitario en tanto:

- Permite a los profesores y al coordinador compartir responsabilidades en todas las fases del proceso de investigación: planificación-acción-reflexión en la búsqueda y aplicación de soluciones a los problemas educativos identificados en la práctica profesional.

- Potencia el desarrollo de la reflexión crítica de los docentes sobre su desempeño profesional y, por tanto, de la tendencia a su autoperfeccionamiento.

En las investigaciones que hemos desarrollado (González Maura, 2000 2003a ) la investigaciónacción-colaborativa ha sido utilizada como modalidad de formación postgraduada en la preparación del profesor universitario para la educación en valores de sus estudiantes durante el proceso de formación profesional en el centro universitario y se ha planteado los siguientes objetivos:

- Diseñar y aplicar estrategias educativas dirigidas a potenciar el desarrollo moral de sus estudiantes en la realización de tareas de aprendizaje en la práctica profesional.

- Valorar la efectividad de las estrategias aplicadas en su contribución al desarrollo moral y profesional del estudiante.

- Reflexionar en torno al desarrollo de su competencia profesional como docente universitario y sobre la efectividad de su función orientadora en la educación moral y profesional de sus estudiantes.

- En el diseño y puesta en práctica de la investigación-acción-colaborativa, como modalidad de formación, hemos tenido en cuenta en sus diferentes momentos: (planificación, acción, reflexión) su contribución al desarrollo profesional del profesor a través de los siguientes aspectos:

\section{En la fase de planificación}

- El diagnóstico de las necesidades de desarrollo profesional de los docentes.

- La sensibilización de los docentes con la necesidad de lograr una actuación profesional autodeterminada en la educación moral y profesional de sus estudiantes.

- La reflexión crítica de su desempeño profesional a través de la identificación de problemas en la educación profesional y en valores de sus estudiantes.

- El compromiso individual y grupal en la toma de decisiones relativas a la elaboración de estrategias educativas dirigidas al desarrollo moral y profesional de sus estudiantes.

En la fase de acción

- El intercambio de experiencias de los docentes en el proceso de puesta en práctica de la estrategia educativa seleccionada.

- La crítica y la autocrítica de la actuación profesional en la puesta en práctica de la estrategia educativa. 
- La flexibilidad en la actuación profesional en la adecuación del plan de acción a las necesidades de los estudiantes y del contexto en el que se desarrolla la estrategia.

- La perseverancia en la búsqueda de solución a los problemas y obstáculos que se presenten durante la aplicación de la estrategia.

En la fase de reflexión

- La reflexión crítica en la valoración de la efectividad de la estrategia educativa aplicada.

- La autonomía en la toma de decisiones que se deriven de la evaluación de la efectividad de la estrategia aplicada.

- El compromiso individual y grupal en el perfeccionamiento de la estrategia aplicada y en el autoperfeccionamiento profesional.

\section{REFLEXIONES FINALES}

Para finalizar y sin pretender hacer conclusiones sobre el tema, quiero apuntar algunas ideas e interrogantes que pueden orientar la reflexión y el debate en torno a la concepción y formación del profesorado universitario a partir de las exigencias de la educación actual.

- El profesor es, ante todo, una persona que como tal se construye en un contexto de interacción social específico: en el ejercicio de su profesión, cuya función esencial es la educación integral de sus estudiantes.

- La función educativa del profesor universitario se expresa a través de su condición de modelo de actuación ética y profesional.

- La investigación-acción constituye un recurso metodológico inestimable en la potenciación del desarrollo profesional del profesor en el ejercicio de la docencia.

- ¿Estamos los profesores universitarios sensibilizados y preparados para ejercer con calidad nuestra función educativa?

\section{REFERENCIAS BIBLIOGRÁFICAS}

BrockBANK, Anne; MC.GILL, Ian (2002) "Aprendizaje reflexivo en la educación superior" Madrid. Morata.

BUXARRAIS, María Rosa (1997) "La formación del profesorado en educación en valores. Propuesta y materiales". Bilbao, Desclée de Brouwer

CANFUX, Verónica. (2000) "La formación psicopedagógica y su influencia en el desarrollo de cualidades del pensamiento del profesor". Tesis de Doctorado. CEPES. Universidad de La Habana.

CORTINA, Adela (1999) "La educación del hombre y del ciudadano". En: "Educación, valores y democracia". Publicación de la OEI, pp.49-74

ELLIOT, John. (1993) "El cambio educativo desde la investigación-acción”. Madrid. Morata.

ESCAMEZ, Juan; GIL, Ramón. (2001) “La educación en la responsabilidad” Barcelona. Paidos.

FREIRE, Paulo (1998) "Pedagogía de la Autonomía" 2da edición. México. Editorial Siglo XXI.. 
GONZÁLEZ MAURA, Viviana. (1999) "El profesor universitario? un facilitador o un orientador en la educación de valores? En: Revista Cubana de Educación Superior. Vol. XIX. (3) pp. 39-48 y en http://www.campus-oei.org/valores/boletin18.htm

GONZÁLEZ MAURA, Viviana. (2001) "La orientación profesional en la educación superior. Una alternativa teórico-metodológica para la formación de profesionales competentes". Monografía. CEPES. Universidad de la Habana.

GONZÁLEZ MAURA, Viviana. (2002) "La profesionalidad del docente universitario desde una perspectiva humanista de la educación". En: Boletín 22. Programa de Educación en Valores. OEl. http://www.campus-oei.org/valores/boletin22.htm y en Monografías Virtuales. Ciudadanía, Democracia y valores en sociedades plurales. (3) http://www.campusoei.org/valores/monografias/monografia03/vivencia01.htm

GONZÁLEZ MAURA, Viviana. (2002a) “ ¿Qué significa ser un profesional competente?” Reflexiones desde una perspectiva psicológica. En: Revista Cubana de Educación Superior. Vol. XII (1) pp. 45-53 y en Revista Iberoamericana de Educación, (versión digital), Sección: De los Lectores. Educación Superior- Educación, trabajo y empleo.

GONZÁLEZ MAURA, Viviana (2003) "Educar valores en la Universidad. Reflexiones desde una perspectiva psicológica." En: Revista Cubana de Psicología. Vol. XX. (1) pp. 50-66

GONZÁLEZ MAURA, Viviana. (2003르) "La investigación-acción-colaborativa como modalidad de formación docente para la educación en valores.". En: "Programa de formación postgraduada para docentes universitarios dirigido a su preparación para la educación en valores". Informe de investigación. CEPES. Universidad de La Habana.

GONZÁLEZ MAURA, Viviana (2004ª $)$ "La formación de la competencia profesional en la Universidad. Reflexiones y experiencias desde una perspectiva educativa". Ponencia. 4to Congreso Internacional de Educación Superior. UNIVERSIDAD 2004 "La Universidad por un Mundo Mejor”. Ciudad de La Habana.

GonZÁLEZ MAURA, Viviana (2004b) " La investigación como eje transversal de la formación postgraduada del docente universitario". Ponencia. 4to Congreso Internacional de Educación Superior. UNIVERSIDAD 2004 "La Universidad por un Mundo Mejor". Ciudad de La Habana.

GonZÁlez Maura,Viviana; BlandeZ,Julia; LóPEZ,Alejandro; SierRA, Miguel Ángel. (2003) "Estrategia educativa y formación docente para el desarrollo del valor responsabilidad en el alumnado de Educación Física". Informe de investigación. Facultad de Educación. Universidad Complutense de Madrid.

GonzÁlez MaURA, Viviana; Rojas Ana Rosa.; CANFuX, Verónica. (2003르) " Principios de un programa de formación postgraduada de docentes universitarios para la educación en valores". Ponencia. III Taller Nacional de investigaciones educativas y Educación de Postgrado. Ministerio de Educación Superior. 7 y 8 de mayo. Ciudad de La Habana.

HERNÁNDEZ, R. A.C. (2000) "Estrategias innovadoras para la formación docente". Desarrollo escolar. Documentos. Biblioteca Digital OEl. http://www.campus-oei.org/oeivirt/ 
IMBERNON, Francisco. (1994) "La formación y el desarrollo profesional del profesorado. Hacia una nueva cultura profesional.". Barcelona. Ed. Grao

IMBERNON, Francisco. (1999) "Conceptualización de la formación y desarrollo profesional del profesorado". En: Ferreres, V y F. Imbernon. "Formación y actualización para la función docente."Madrid. Editorial Síntesis. Pp.. 25-34

IMBERNON, Francisco. (2001) "La profesión docente ante los desafíos del presente y del futuro". En: Marcelo, C. (editor) "La función docente". Madrid. Editorial Síntesis. pp. 27-41

IMBERNON, Francisco. (2002) (coord) "La investigación educativa como herramienta de formación del profesorado". Barcelona. Editorial Graó

KRAFTCHENKO, Oksana y SEGARTE, Ana Luisa (1999) "Diplomado en Rol profesional y tarea educativa" Informe de Investigación. CEPES. Universidad de La Habana.

HoYos, Guillermo (1999) "Ética comunicativa y educación para la democracia". En "Educación, valores y democracia”. Publicación de la OEI, p9-47

KEMMIS, Stephen; MC TAGGART, Robin (1992) ¿Cómo planificar la investigación-acción? Barcelona. Editorial Laertes.

MARTín, Elvira; GonZÁleZ, Miriam y GonZÁlez MAURA, Viviana (2002) "Experiencias en el apoyo a la formación de profesores de la Educación Superior". Revista Tarbiya. (30). 1er semestre. Universidad Autónoma de Madrid. pp. 63-77

MARTíneZ, Miquel (2000) "El contrato moral del profesorado. Condiciones para una nueva escuela. " 2da edición, Bilbao, Desclée de Brouwer

MaRTíneZ, Miquel; BuXARRAIS, María Rosa; Esteban, Francisco (2002) "La universidad como espacio de aprendizaje ético". Revista Iberoamericana de Educación, No. 29, pp. 17-43

MUÑOZ, J. QUINTERO y J. MUNEVAR, (2002) "Experiencias en investigación-acción-reflexión con educadores en proceso de formación en Colombia. Revista Electrónica de Investigación Educativa. Vol 4.(1) . http://redie.ens.uabc.mx/vol4no1/contenido-munevar.html

NAVARRO, Ginés (2000) “El diálogo. Procedimiento para educar en valores” Bilbao, Desclée de Brouwer

OROzco, D. (2000) "Experiencia de capacitación en Costa Rica. Perfiles de los educadores y educadoras y la formación permanente de cara al nuevo milenio". Ministerio de Educación Pública. Centro Nacional de Didáctica. En : http://www.campus-oei.org/oeivirt/ Biblioteca Digital.

OJALVO, et. al (2003) "Programa de formación postgraduada de docentes universitarios para la educación en valores desde el curriculum". Informe de investigación. CEPES. Universidad de La Habana.

PINE, G. (1981): “Colaborative action research. The integration of research And Service". Paper presented at the annual meeting of American Asociation of Colleges for teaching education. Detroit.

RODRíGUEZ, J. G. (1999) "Desarrollo educativo y formación del profesorado. La experiencia del programa RED en la Universidad Nacional de Colombia. "Desarrollo Escolar. I Seminario Taller sobre perfil del docente y Estrategias de formación. Biblioteca Digital. OE/ http://www.campus-oei.org/oeivirt/ 
SÁNCHEZ MUÑOZ, José Antonio. (2002) "La formación inicial para la docencia universitaria. Biblioteca Digital. OEl. http://www.campus-oei.org/oeivi rt/

UNESCO (1998) "La Educación Superior en el Siglo XXI: Visión y Acción". Conferencia Mundial sobre la Educación Superior. París, 5 -9 de octubre.

VINUESA, María del Pilar. (2002) "Construir los valores. Curriculum con aprendizaje cooperativo". " Bilbao, Desclée de Brouwer 


\title{
Contactar
}

Revista lberoamericana de Educación

\author{
Principal OEI
}

